\title{
An Apple microcomputer system in physiological psychology
}

\author{
ANTHONY G. ROMANO, JOSEPH E. STEINMETZ, and MICHAEL M. PATTERSON \\ College of Osteopathic Medicine, Ohio University, Athens, Ohio
}

\begin{abstract}
The use of microcomputers in physiological psychology has allowed many investigators to conduct experiments that previously required more costly devices. We describe some of the research requirements that led to our selection of an Apple II/FIRST microcomputer system (Scandrett \& Gormezano, 1980) for investigations of the neurophysiological correlates of classical and instrumental conditioning.
\end{abstract}

Physiological psychology has been drastically affected by the microcomputer revolution. Microcomputers have made feasible experiments that were either extremely laborious or impossible only a few years ago. In this paper, we outline how we are using microcomputers to perform tasks in brain-behavior studies.

Our research is concerned with delineating the neurophysiological correlates of classical and instrumental conditioning. This research entails the use of lesion, stimulation, and electrophysiological techniques, along with a variety of conditioning paradigms. Several years ago, we made the transition from the use of electronic modules, oscilloscopes, and polygraphs to an Apple II/FIRST microcomputer system (Scandrett \& Gormezano, 1980) for data collection and control of experiments. Some of the considerations which led to the selection of this particular system and some of the applications that we have made are described in this paper.

The ability to perform tasks in real time was the most important factor we considered. For our experiments, real-time tasks are segregated into two broad classes: data acquisition and stimulus control. Clearly, the ability to perform real-time data acquisition depends on the nature of the data to be quantified. The response systems measured in our experiment include nictitating membrane (NM) responses of cats or rabbits and hindlimb-flexion responses of cats. Each type of response consists of a relatively discrete movement. Typical unconditioned responses have latencies in the range of $25-50 \mathrm{msec}$ and durations from $150-300 \mathrm{msec}$. The minimum response amplitude of interest is traditionally $0.5 \mathrm{~mm}$ of $\mathrm{NM}$ or leg movement. Maximum response amplitudes are generally less than $16 \mathrm{~mm}$ for NM responses and about twice

The hardware and software described in this report were supported in part by the Ohio University College of Osteopathic Medicine, American Osteopathic Association Bureau of Research Grant 81-80, and NIH Grant 2-14545.

J. E. Steinmetz is currently at the Department of Psychology, Building 420/Jordan Hall, Stanford University, Stanford, CA 94305.

Requests for reprints should be sent to Michael M. Patterson, College of Osteopathic Medicine, Ohio University, Athens, OH 45701-2979. that for leg-flexion responses. Polygraph tracings of representative cat NM and leg-flexion responses are shown in Figures 1 and 2. Numerous conditioning studies that have employed these and other skeletal response systems have shown that conditioning procedures affect several topographical features of these responses, such as onset latency, peak latency, peak amplitude, and the area under the response.

In addition to monitoring skeletal response systems in intact preparations, some of our studies employ spinalized cats in which conditioning is indexed by a change in the magnitude of a motor nerve response. This response is composed of summated action potentials elicited by stimulation of a sensory nerve. The topography of the response differs considerably from the skeletal responses described above. Response latency is generally 8-10 msec, response duration is $2-3 \mathrm{msec}$, and amplitude is in the range of $20-60 \mu \mathrm{V}$. We also monitor neuronal unit activity from a variety of central nervous system sites in all of the preparations. Figure 3 is an oscilloscope tracing of unit activity recorded from the hippocampus

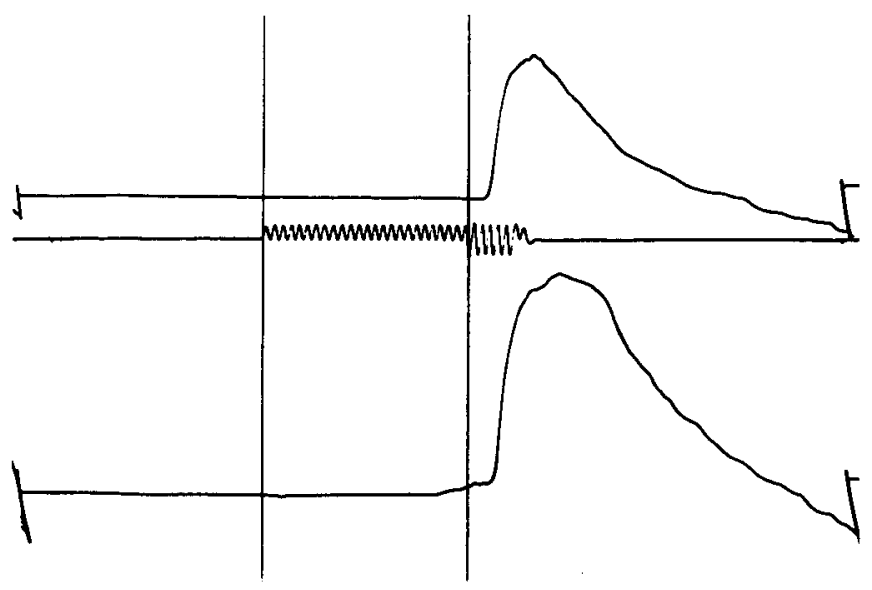

Figure 1. Polygraph tracings of cat nictitating membrane (NM) responses. The two vertical lines mark the onsets of the conditioned and unconditioned stimuli. The upper tracing shows only the unconditioned response; the lower tracing shows a conditioned response beginning shortly before the onset of the unconditioned stimulus. 


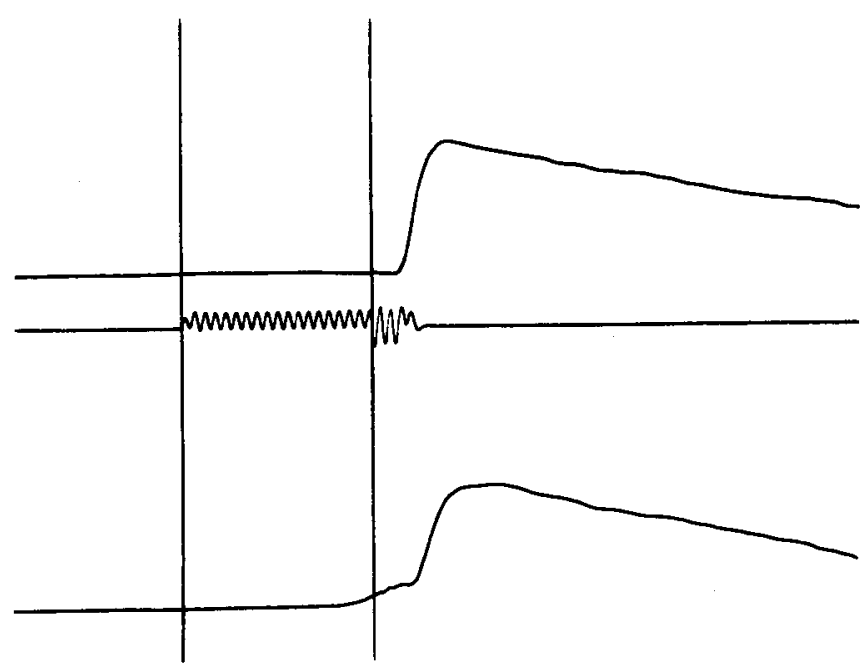

Figure 2. Polygraph tracings of cat leg-flexion responses. As in Figure 1, the upper tracing shows only an unconditioned response, and the lower tracing shows both conditioned and unconditioned responses.

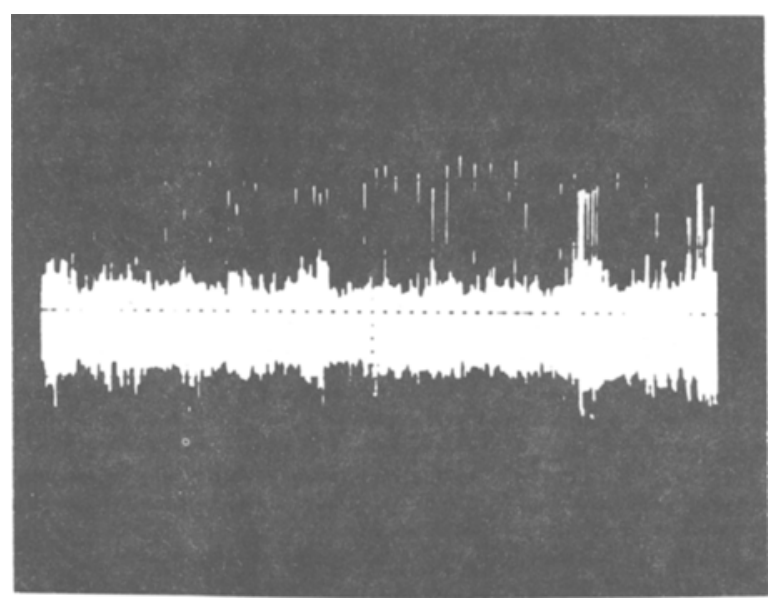

Figure 3. Oscilloscope tracing of multiple-unit hippocampal activity. Trace duration is 0.5 sec.

of a rabbit. Depending upon the recording site, unit activity varies between several spikes per second to several hundred spikes per second and is traditionally quantified as frequency per unit time and/or interspike interval. Both measures are of interest for conditioning studies, as is the correlation between unit activity and the response being conditioned.

The Apple II/FIRST system which we employ for intact preparations is illustrated in Figure 4. The system consists of a 16-channel, 8-bit A/D converter; a 3-channel, 16-bit counter/timer; and several 24-bit programmable peripheral interfaces. The A/D converter is used for online acquisition of behavioral data. The rate of conversion is determined by the counter/timer. Typically, a conversion is performed every $5 \mathrm{msec}$. The counter/timer also is used for intertrial and stimulus timing, as well as for neuronal spike counting. The peripheral interface devices are used for enabling and disabling external relay drivers which gate the stimuli on and off. The buffer amplifiers interposed between the NM signal and the A/D converter allow voltage level adjustments to be made to each signal before it reaches the A/D converter. Thus, we can compensate for shifts in the position of the NM and thereby maintain an optimal input range for the $A / D$ converter. The buffer amplifiers, which also determine the sensitivity of the A/D converter to NM or leg movement, are currently set to produce a change of 16 digital counts for every $1 \mathrm{~mm}$ of NM movement or 8 counts for every $1 \mathrm{~mm}$ of hindlimb movement.

During a typical NM conditioning session, multiple unit neuronal activity is recorded on magnetic tape, and the digitized NM response is stored on floppy disk. Although the flexibility and speed of the FIRST language would allow us to count neuronal spikes and digitize behavioral data simultaneously, we have elected to dedicate the system only to on-line acquisition of behavioral data and to count neuronal spikes off-line. Thus, for each trial, a timing signal is recorded on tape to mark the start of the trial. The first $500 \mathrm{msec}$ of the trial consist of a baseline period during which the NM data is digitized every $5 \mathrm{msec}$. At the end of this period, a machine-language routine computes the average of the baseline counts. Each digital count obtained during the baseline period is then compared with this average. Any deviation in excess of $0.25 \mathrm{~mm}$ of NM movement restarts the baseline period, and the entire process is repeated. Once a stable baseline is obtained, a second timing signal is recorded on tape to mark the end of the baseline period and the onset of the conditioned stimulus. The baseline procedure serves two purposes: It ensures (1) that NM movement during the trial will be compared against a stable baseline and (2) that baseline neuronal activity will not be contaminated by gross movement artifacts or spontaneous NM movements. For instrumental conditioning, additional real-time control is required. Thus, for an avoidance paradigm, as soon as the conditioned stimulus is presented, each incoming A/D count is compared with the average computed during the baseline period. Any deviation corresponding

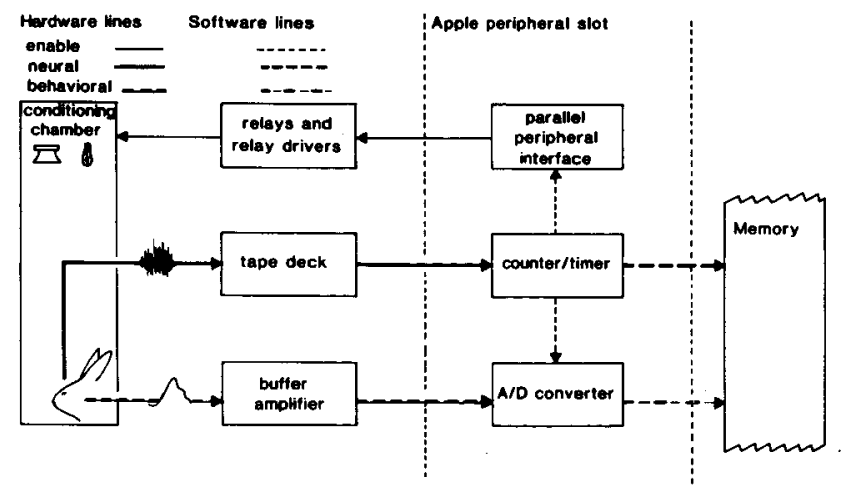

Figure 4. Block diagram of the Apple II/FIRST system used for skeletal response conditioning. See text for details. 


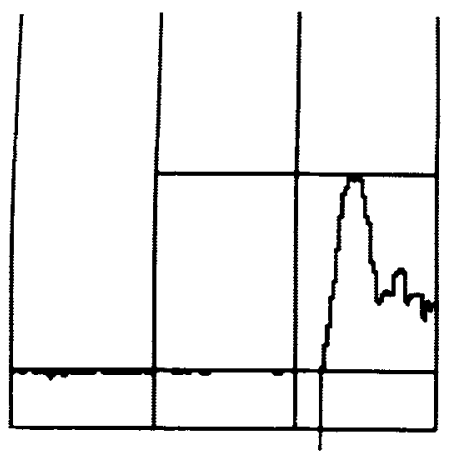

\section{$L A T=295 \quad A M P=61$}

PKLAT $=345 \quad B A S=211$

AREA $=113$ RMS $=48$

sijB.j 1

TRIAL $=77$ :

Figure 5. Computer display of rabbit NM response, grid overlay of trial events, and measures of NM response topography.

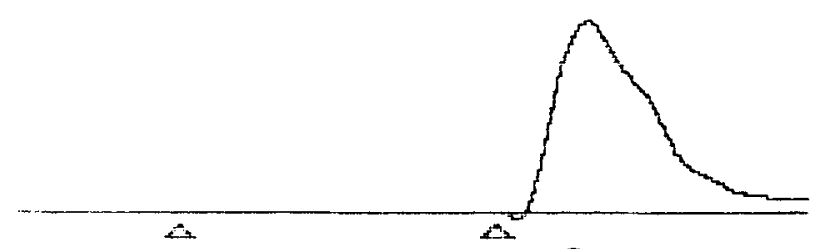

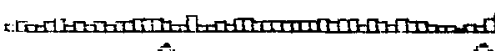

Figure 6. Average NM response and histogram of summed hippocampal activity for one conditioning session. The first and second cursors mark the onsets of the conditioned and unconditioned stimuli, respectively. to our preestablished definition of a conditioned response resets the output bit normally used to turn on the unconditioned stimulus. Thus, the animal avoids the unconditioned stimulus.

At the end of each trial, the array of digitized NM data is written to disk and a graph of the NM response is displayed on the monitor screen, as shown in Figure 5. The grid overlay marks the trial events. The left vertical line marks the beginning of the baseline period, the next two lines mark the onset of the conditioned and unconditioned stimuli, respectively, and the last line marks the end of the trial. The data below the grid are measures of response topography.

Off-line neuronal spike counting is accomplished by playing the tape-recorded signal through a window discriminator and using one channel of the counter/timer to count the output pulses of the discriminator. The remaining channels of the counter/timer are used in the same modes as those used during the conditioning session. The software polls the tape recorder for the prerecorded timing signals, and spike counts are cumulated into 5 -msec time bins. The array of data for each trial is then stored on a floppy disk. After the data have been collected, an overall picture of the correlation between brain activity and behavior is obtained by averaging the NM response over trials and summing the neural activity over trials into 15-msec time bins. A representative NM response and a histogram of hippocampal activity is shown in Figure 6. Finer-grained analyses of the neurobehavioral correlations are also performed for blocks of trials within a conditioning session. These analyses typically correlate brain activity with behavior by summing or averaging the data in 5-msec time bins over a block of 10 trials. Figure 7 is an example of the average NM response and cumulated neural activity for one block of trials. Note that neural activity appears to increase prior to the onset of the be-

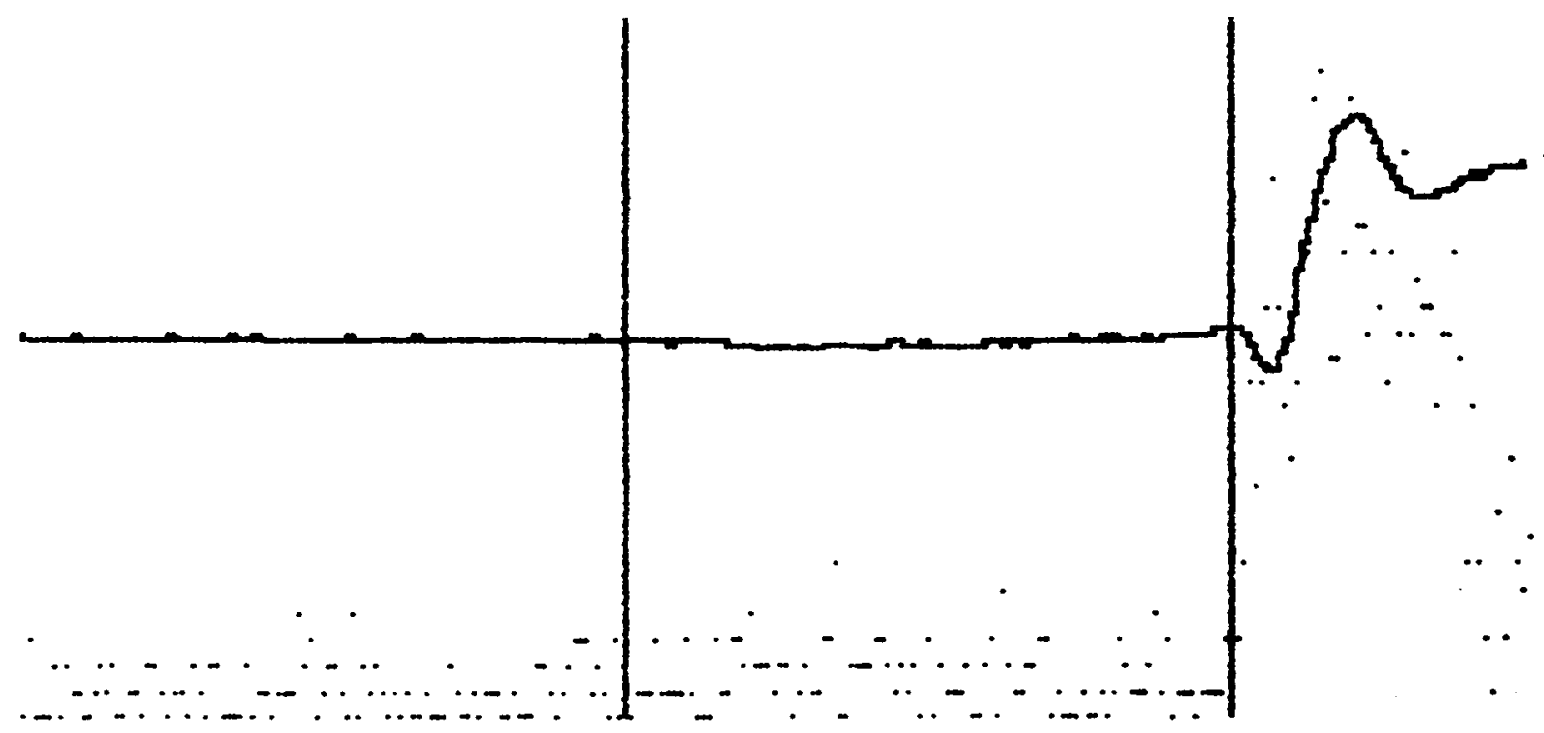

Figure 7. Average NM response and summed unit activity for a single block of trials. Note the temporal asynchrony between unit activity and behavior. 
havioral response. Because this temporal asynchrony tends to degrade the neurobehavioral correlation, cross-correlations are computed by repeatedly shifting the neural activity forward by one bin at a time and recorrelating the data. The results of such an analysis are shown in Figure 8. In this particular case, the neurobehavioral correlation is maximal when neural activity precedes the behavioral response by about $50-55 \mathrm{msec}$.

It is worth noting that, during each stage of data reduction, the experimenter never transcribes a single data point. All of our software operates directly on the data disks, thus virtually eliminating the possibility of a transcription error.

The Apple II/FIRST system used for the spinal preparation is illustrated in Figure 9. The major difference between this system and the one described above is the A/D converter. Because the motor nerve response exhibits a much shorter latency and duration than the skeletal responses, a higher-speed A/D converter is required for accuracy. Furthermore, the converter must run at a rate independent of that used for stimulus timing. The conversion

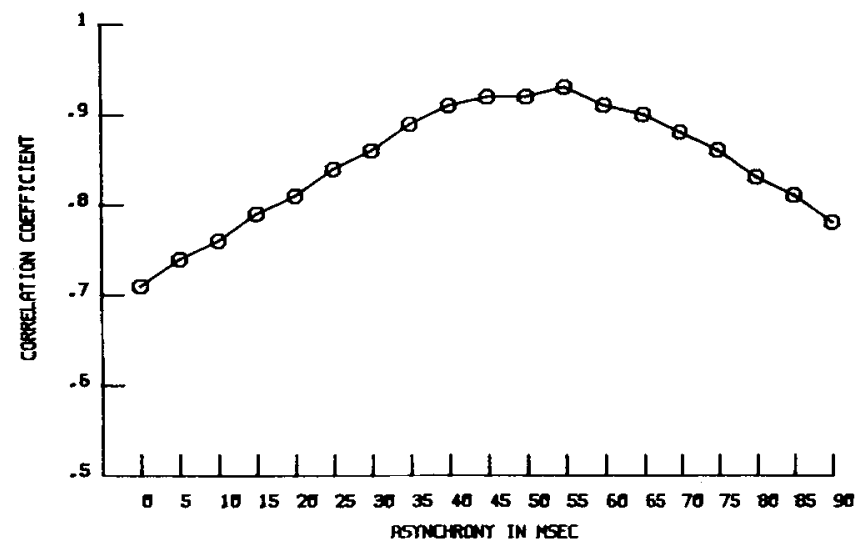

Figure 8. Graph of the cross-correlations obtained from the data depicted in Figure 7.

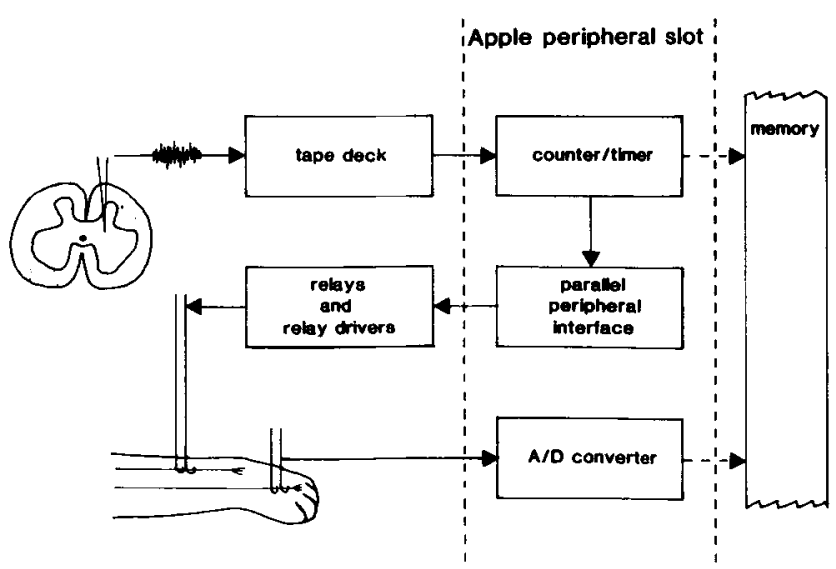

Figure 9. Block diagram of the Apple II/FIRST system used for spinal conditioning. See text for details.

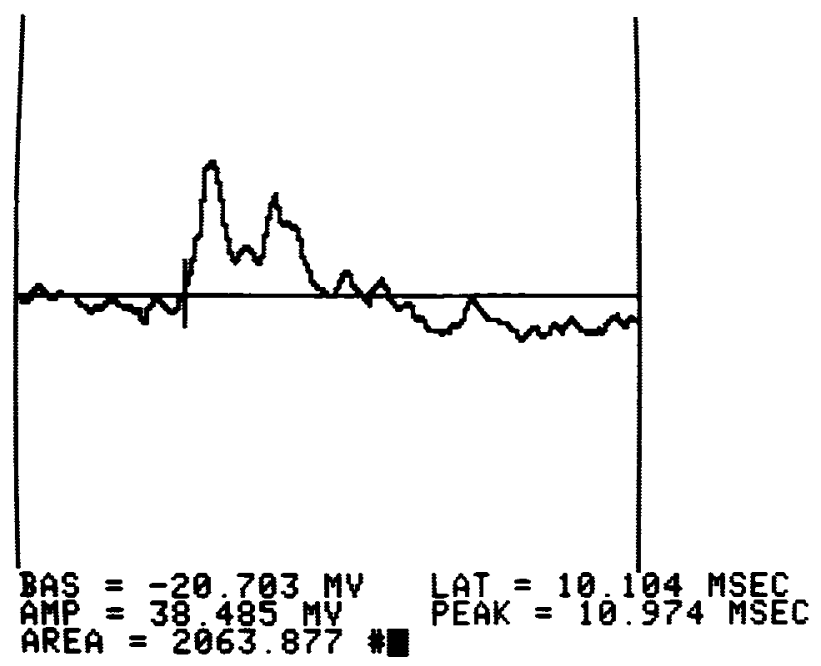

Figure 10. Computer display of motor nerve response and measures of response topography.

rate is therefore under the control of a software machine language routine that yields a conversion rate of about $17 \mathrm{kHz}$. All other features of the two systems are the same. The conditioning data are obtained on-line and neuronal unit activity is recorded on tape for subsequent off-line spike counting. Again, at the completion of each trial, the digitized data are stored on disk, and the response is displayed on the monitor screen as shown in Figure 10. The width of the display represents a duration of about $15 \mathrm{msec}$, beginning with the onset of the conditioned stimulus. The response itself is composed of 400 successive A/D conversions.

The power of the Apple II/FIRST system as a research tool should be evident in the previous description. The system has given us the ability to explore the details of brain-behavior relationships using techniques that previously required costly minicomputers or mainframes. As one example, we now routinely compute cross-correlations between neural activity and the waveform of a behavioral response. The procedure typically computes 20 cross-correlations using 150 pairs of data points for each of 10 blocks of trials and is completed within minutes after the raw data have been collected. Clearly, the use of such fine-grained descriptions of brain-behavior relationships will lead to finer interpretations of brain function. Similarly, the ability to easily quantify conditioned changes in the topographical features of behavioral responses is likely to lead to finer theoretical treatments of conditioning. In the past, the tedious measurement of response topography has led most researchers to describe conditioned changes in behavior only in terms of response frequency. However, trial-by-trial changes in response topography may provide more accurate indices of learning, as well as a broader data base for testing and refining theories of conditioning. Finally, the routine measurement of response topography will provide the means to readily 
detect the subtle changes in behavior which often accompany experimentally induced brain damage. Indeed, we have already reported data in which such changes are evident (Port, Mikhail, \& Patterson, 1985).

In summary, the Apple II/FIRST system is an extremely powerful research tool. It currently provides a costefficient and versatile means of conducting neurophysiological investigations of conditioning.

\section{REFERENCES}

Port, R. L., Mikhail, A. A., \& Patterson, M. M. (1985). Differential effects of hippocampectomy on classically conditioned nictitating membrane response related to interstimulus interval. Behavioral Neuroscience, 99, 200-208.

SCANDRETT, J., \& GoRmezano, I. (1980). Microprocessor control and A/D data acquisition in classical conditioning. Behavior Research Methods \& Instrumentation, 12, 120-125. 